\title{
Existence of Multiple Solutions for Second-Order Problem with Stieltjes Integral Boundary Condition
}

\author{
Qing-Qing Hu $(\mathbb{D}$ and Baoqiang Yan \\ School of Mathematics and Statistics, Shandong Normal University, Jinan 250014, China \\ Correspondence should be addressed to Baoqiang Yan; yanbqcn@aliyun.com
}

Received 24 December 2020; Revised 15 January 2021; Accepted 9 February 2021; Published 25 March 2021

Academic Editor: Maria Alessandra Ragusa

Copyright (c) 2021 Qing-Qing Hu and Baoqiang Yan. This is an open access article distributed under the Creative Commons Attribution License, which permits unrestricted use, distribution, and reproduction in any medium, provided the original work is properly cited.

In this paper, we consider the existence of multiple solutions for second-order equation with Stieltjes integral boundary condition using the three-critical-point theorem and variational method. Firstly, a novel space is established and proved to be Hilbert one. Secondly, based on the above work, we obtain the existence of multiple solutions for our problem. Finally, in order to illustrate the effectiveness of our problem better, the example is listed.

\section{Introduction}

We are concerned with the following general second-order differential equation

$$
\left\{\begin{array}{l}
-\omega^{\prime \prime}(t)=\beta g(t, \omega(t)), \\
\omega(0)=0, \omega(1)=\int_{0}^{1} \omega(s) d \gamma(s),
\end{array}\right.
$$

where $\beta$ is a positive parameter, $g:[0,1] \times \mathbb{R} \longrightarrow \mathbb{R}$ is a continuous function, and $\gamma(s)$ is a nondecreasing function, $0<\int_{0}^{1} s d \gamma(s)<1,0<\int_{0}^{1} s^{2} d \gamma(s)<1$.

In the past few decades, the boundary value problems have appealed to many scholars in the mathematical field. Generally speaking, the boundary value problems mostly involve in two-point [1-5], three-point [6-8], and multipoint [9-11]. Many physical phenomena were formulated as nonlocal mathematical models with integral boundary conditions [12-21], such as fractional differential equation [22-30], nonlinear singular parabolic equation [31], and general second-order equation [19, 32-40].

For the integral boundary value problems such as (1), many researchers studied mainly by the method of topologi- cal degree. For examples, using the fixed point theorem, Ma [39] studied ordinary second-order equation as below

$$
\left\{\begin{array}{l}
\chi^{\prime \prime}+a(t) g(\chi)=0,0<t<1, \\
\chi(0)=0, \chi(1)=\int_{\xi}^{\eta} \gamma(z) \chi(z) d z,
\end{array}\right.
$$

where $0<\xi<\eta<1$ and $g(\chi)$ are either superlinear or sublinear and obtained the existence of positive solutions; Karakostas et al. [19] studied the existence of three positive solutions of the following problem

$$
\left\{\begin{array}{l}
\chi^{\prime \prime}+\chi(t) g(\chi)=0,0 \leq t \leq 1, \\
\chi(0)=0, \chi(1)=\int_{\xi}^{\eta} \chi(z) d \gamma(z)
\end{array}\right.
$$

by the theory of fixed point index on a cone; Benchohra et al. [33] investigated the following second-order equation

$$
\left\{\begin{array}{l}
\chi^{\prime \prime}+g(t, \chi)=0,0 \leq t \leq 1, \\
\chi(0)=0, \chi(1)=\int_{0}^{1} \chi(z) \gamma(z) d z
\end{array}\right.
$$


via contraction principle and Leray-Schauder alternative theorem; Galvis [41] considered the nonlinear second-order problem

$$
\left\{\begin{array}{l}
\chi^{\prime \prime}+\chi(t) g(t, \chi)=0,0 \leq t \leq r, \\
\chi(0)=0, \chi(r)=\beta \int_{0}^{a} \chi(z) d z
\end{array}\right.
$$

by Schauder's fixed point theorem. Some other works on fractional equation with integral boundary conditions can be found in $[26,28,29]$.

To the best of my knowledge, no one use the theory of critical point and variational method to deal with the existence of solution of problem (1).

In order to discuss problem (1), we introduce a new space as follows:

$$
\mathscr{W}(0,1)=\left\{\omega \in W^{1,2}(0,1): \omega(0)=0, \omega(1)=\int_{0}^{1} \omega(s) d \gamma(s)\right\}
$$

endowed with the norm $\|\omega\|=\left[\int_{0}^{1}\left|\omega^{\prime}\right|^{2} d t\right]^{1 / 2}$.

The paper is organized as follows. In Section 2, we introduce some concepts related to solve problem (1) more conveniently. In Section 3, we prove that constructed space is a Hilbert. Section 4 demonstrates the existence of at least three solutions for problem (1) mostly via a three-critical-point theorem. Section 5 gives an example to explain efficacy of our method. Our method is different from those in $[19,33$, $39,41]$, and the nonlinear term $g$ is neither superlinear nor sublinear in our paper. Some ideas of our proof come from $[42,43]$.

\section{Preliminaries}

Definition 1. Let $(\bar{E}, \varsigma)$ and $\left(\bar{E}_{1}, \varsigma_{1}\right)$ be metric spaces, and the operator $\widetilde{\phi}: \bar{E} \longrightarrow \bar{E}_{1}$ meets the conditions as follows:

(1) The mapping $\widetilde{\phi}$ is surjective

(2) $\varsigma(\omega, v)=\varsigma_{1}(\widetilde{\phi} \omega, \widetilde{\phi} v)$, for any $\omega, v \in \bar{E}$

Then, $(\bar{E}, \varsigma)$ is isometric isomorphic to $\left(\bar{E}_{1}, \varsigma_{1}\right)$; moreover, $\widetilde{\phi}$ is named as an isometric isomorphic mapping.

Lemma 2. Let $\bar{E}$ be linear normed space. Suppose that for any $\tilde{\varepsilon} \in(0,2)$, there exist $\tilde{\delta}=\tilde{\delta}(\tilde{\varepsilon})>0,\|\omega\|+\|v\|=1,\|\omega-v\| \geq \varepsilon$ satisfying

$$
\left\|\frac{\omega+v}{2}\right\| \leq 1-\tilde{\delta}(\tilde{\varepsilon})
$$

then, space $\bar{E}$ is uniformly convex.

Lemma 3 (Milman theorem [44]). A uniformly convex Banach space is reflexive.
Lemma 4 (Clarkson inequality [44]). For $\omega, v \in L^{p}((0,1)), 2$ $\leq q<+\infty$, one has

$$
\left\|\frac{\omega+v}{2}\right\|^{p}+\left\|\frac{\omega-v}{2}\right\|^{p} \leq \frac{\|\omega\|^{p}+\|v\|^{p}}{2}
$$

Lemma 5 ([45]). Let $\mathscr{X}$ be a nonempty set. $Y$ and $\Phi$ are two real functions on $\mathscr{X}$. Assume that there are $\alpha>0$ and $\omega_{0}, \omega_{1}$ $\in \mathscr{X}$ such that

$$
\begin{gathered}
Y\left(\omega_{0}\right)=\Phi\left(\omega_{0}\right)=0, \\
Y\left(\omega_{1}\right)>\alpha, \\
\sup _{\omega \in Y^{-1} \ddot{£}(-\infty, \alpha] £ \odot} \Phi(x)<\alpha \frac{\Phi\left(\omega_{1}\right)}{Y\left(\omega_{1}\right)} ;
\end{gathered}
$$

then, for all $\rho$, satisfying

$$
\sup _{\omega \in Y^{-1} \hat{\mathscr{E}}(-\infty, \alpha] \in \odot} \Phi(x)<\rho<\alpha \frac{\Phi\left(\omega_{1}\right)}{Y\left(\omega_{1}\right)}
$$

one has

$\sup _{\beta \geq 0} \inf _{\omega \in \mathscr{X}}\left(Y(\omega)+\beta(\rho-\Phi(\omega))<\inf _{\omega \in \mathscr{X}} \sup _{\beta \geq 0}(Y(\omega)+\beta(\rho-\Phi(\omega))\right.$

Theorem 6 (three-critical-point theorem [46]). Let $\mathscr{X}$ be a

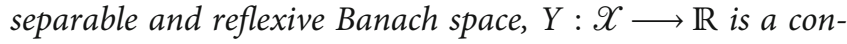
tinuously Gâteaux differentiable and sequentially weakly lower semicontinuous functional whose Gâteaux derivative generates a continuous inverse on $\mathscr{X}^{*}$, and $\Phi: \mathscr{X} \longrightarrow \mathbb{R}$ is a continuously Gâteaux differentiable function whose Gâteaux derivative is compact. Assume

(1) for all $\beta \in[0,+\infty)$,

$$
\lim _{\|\omega\| \rightarrow+\infty} Y(\omega)+\beta \Phi(\omega)=+\infty
$$

(2) there exists a continuous concave function $H:[0,+\infty)$ $\longrightarrow \mathbb{R}$ such that

$$
\sup _{\beta \geq 0} \inf _{\omega \in \mathscr{X}}(Y(\omega)+\beta \Phi(\omega)+H(\beta))<\inf _{\omega \in \mathscr{X}} \sup _{\beta \geq 0}(Y(\omega)+\beta \Phi(\omega)+H(\beta))
$$

Then, there exist an open interval $\Omega \subseteq(0,+\infty)$ and $a$ positive real number $p$ such that, for each $\beta \in \Omega$, the equation

$$
Y^{\prime}(\omega)+\beta \Phi^{\prime}(\omega)=0
$$

has at least three solutions in $\mathscr{X}$ whose norms are less than $p$. 


\section{Separability and Reflexivity for Space $\mathscr{W}$}

In this section, we illustrate that $\mathscr{W}$ is a separable and reflexive real Banach space to guarantee our main results. The following theorem is given.

Theorem 7. W is a separable and reflexive real Banach space.

Proof. The proof is divided into three parts.

Part 1. Space $\mathscr{W}$ is a Banach space.

It is well known that $\mathscr{W}$ is a normed linear space. Let $\left\{\omega_{n}(x)\right\}$ be an arbitrary cauchy sequence of space $\mathscr{W}$. According to Morrey's inequality [47], one has

$$
\left\|\omega_{n}(x)-\omega(x)\right\|_{\mathscr{C}^{0, r}} \leq M_{0}\left\|\omega_{n}(x)-\omega(x)\right\|_{\mathscr{W}},
$$

where $M_{0}$ is a constant, $r \in(0,1)$. So, for any $n, \omega_{n}(x)$ is uniform convergence in space $\mathscr{W}$, which means

$$
\left\{\begin{array}{l}
\omega_{n}(0)=\omega(0)=0 \\
\lim _{n \rightarrow+\infty} \omega_{n}(1)=\omega(1)
\end{array}\right.
$$

By Lebesgue control convergence theorem, we have

$\lim _{n \rightarrow+\infty} \omega_{n}(1)=\lim _{n \rightarrow+\infty} \int_{0}^{1} \omega_{n}(s) d \gamma(s)=\int_{0}^{1} \lim _{n \rightarrow+\infty} \omega_{n}(s) d \gamma(s)=\int_{0}^{1} \omega(s) d \gamma(s)$.

From the second of (16) and (17), we obtain $\omega(1)=\int_{0}^{1} \omega$ $(s) d \gamma(s)$. Therefore, Part 1. holds.

Part 2. Space $\mathscr{W}$ is separable.

Let $\Lambda \subset W^{1,2}(0,1)$ be an enumerable subset. Due to the separability of space $W^{1,2}(0,1)$, there exists $\left\{w_{n}\right\} \subset \Lambda$ with $\lim _{n \rightarrow+\infty} w_{n}=w$, for any $w \in W^{1,2}(0,1)$.

Define mapping $\mathscr{T}: \Lambda \longrightarrow \mathscr{W}$ satisfying

$$
\begin{aligned}
(\mathscr{T} w)(t)= & w(t)+\frac{1}{M_{1}}\left(-\widehat{A} t^{2}+t\right)\left[\int_{0}^{1} w(s) d \gamma(s)-y(1)\right] \\
& -\left(M_{2} t^{2}+M_{3} t+1\right) w(0)
\end{aligned}
$$

where $w \in \Lambda$. Constants $\widehat{A}, M_{1}, M_{2}$, and $M_{3}$ in (3) are denote by

$$
\left\{\begin{array}{l}
\widehat{A}=\frac{1-\int_{0}^{1} t d \gamma(t)}{1-\int_{0}^{1} t^{2} d \gamma(t)} \\
M_{1}=\frac{\int_{0}^{1} t d \gamma(t)-\int_{0}^{1} t^{2} d \gamma(t)}{1-\int_{0}^{1} t^{2} d \gamma(t)} \\
M_{2}=\frac{\int_{0}^{1} d \gamma(t)-\int_{0}^{1} t d \gamma(t)}{\int_{0}^{1} t d \gamma(t)-\int_{0}^{1} t^{2} d \gamma(t)} \\
M_{3}=\frac{\int_{0}^{1} t^{2} d \gamma(t)-\int_{0}^{1} d \gamma(t)}{\int_{0}^{1} t d \gamma(t)-\int_{0}^{1} t^{2} d \gamma(t)}
\end{array}\right.
$$

Let $\Theta=\mathscr{T}(w)$, and it is easy to obtain $\Theta \in \mathscr{W}$. Consider set $\widehat{\Lambda}=\{M=\mathscr{T}(w): w \in \Lambda\} ;$ so, $\widehat{\Lambda} \subset \mathscr{W}$ is an enumerable subset.

Next, let us demonstrate that there exists $M_{n} \subset \widehat{\Lambda}$ with $\lim _{n \rightarrow+\infty} M_{n}=w$, for any $w \in \mathscr{W}$. Owing to $w \in \mathscr{W}$, there exists sequence $\left\{w_{n}\right\} \subset \Lambda$ such that $\lim _{n \rightarrow+\infty} w_{n}=w$. Let $M_{n}$ $=\mathscr{T}\left(w_{n}\right)$ and write

$$
\begin{aligned}
& \left\|M_{n}-w_{n}\right\|=\left[\int_{0}^{1}\left|\left(M_{n}-w_{n}\right)^{\prime}\right|^{2} d t\right]^{1 / 2} \\
& =\left[\int _ { 0 } ^ { 1 } \left(\frac{1}{M_{1}}(-2 \widehat{A} t+1)\left(\int_{0}^{1} \omega_{n}(s) d \gamma(s)-\omega_{n}(1)\right)\right.\right. \\
& \left.\left.-\left(2 M_{2} t+M_{3}\right) \omega_{n}(0)\right)^{2} d t\right]^{1 / 2} \\
& =\left[\int _ { 0 } ^ { 1 } \left(\frac{1}{M_{1}^{2}}(-2 \widehat{A} t+1)^{2}\left(\int_{0}^{1} \omega_{n}(s) d \gamma(s)-\omega_{n}(1)\right)^{2}\right.\right. \\
& +\left(2 M_{2} t+M_{3}\right)^{2} \omega_{n}^{2}(0)-2 \frac{1}{M_{1}}(-2 \widehat{A} t+1) \\
& \left.\left.\cdot\left(2 M_{2} t+M_{3}\right)\left(\int_{0}^{1} \omega_{n}(s) d \gamma(s)-\omega_{n}(1)\right) \omega_{n}(0) d t\right)\right]^{1 / 2} \\
& =\left[\left(\frac{4 \widehat{A}^{2}}{3}+1-2 \widehat{A}\right)\left(\int_{0}^{1} \omega_{n}(s) d \gamma(s)-\omega_{n}(1)\right)^{2}\right. \\
& +\left(\frac{4 M_{2}^{2}}{3}+M_{3}^{2}+2 M_{2} M_{3}\right) \omega_{n}^{2}(0)-\frac{2}{M_{1}}\left(-\frac{4 \widehat{A} M_{2}}{3}\right. \\
& \left.\left.-\widehat{A} M_{3}+M_{2}+M_{3}\right)\left(\int_{0}^{1} \omega_{n}(s) d \gamma(s)-\omega_{n}(1)\right) \omega_{n}(0)\right]^{1 / 2} \\
& \rightarrow\left[\left(\frac{4 \widehat{A}^{2}}{3}+1-2 \widehat{A}\right)\left(\int_{0}^{1} \omega(s) d \gamma(s)-\omega(1)\right)^{2}\right. \\
& +\left(\frac{4 M_{2}^{2}}{3}+M_{3}^{2}+2 M_{2} M_{3}\right) \omega^{2}(0)-\frac{2}{M_{1}}\left(-\frac{4 \widehat{A} M_{2}}{3}\right. \\
& \left.\left.-\widehat{A} M_{3}+M_{2}+M_{3}\right)\left(\int_{0}^{1} \omega(s) d \gamma(s)-\omega(1)\right) \omega(0)\right]^{1 / 2} \\
& =0, \text { as } n \rightarrow+\infty \text {. }
\end{aligned}
$$

Hence, Part 2. holds.

Part 3. $\mathscr{W}$ is reflexive.

Define operator $\mathscr{P}$ :

$$
\mathscr{W} \longrightarrow L^{2}(0,1)
$$

That is to say, $\mathscr{P}: \omega \longrightarrow \omega^{\prime} \in L^{2}(0,1)$, for all $\omega \in \mathscr{W}$. Let set

$$
Y=\left\{\omega^{\prime}: \omega \in \mathscr{W}\right\}
$$

endowed with the norm

$$
\|v\|=\sqrt{\int_{0}^{1}|v|^{2} d t}
$$


we obtain

$$
Y \subset L^{2}(0,1)
$$

Consequently,

$$
Y \subset L^{2}(0,1), \quad \omega^{\prime}=v
$$

Next, we will prove operator $\mathscr{P}$ is an isometric isomorphic mapping. Evidently, mapping $\mathscr{P}$ is surjective. By Definition 1 , we only illustrate $\mathscr{P}$ as isometric. According to mapping $\mathscr{P}: \mathscr{W} \longrightarrow Y$, we deduce

$$
\|\omega\|=\|v\|=\|\mathscr{P} \omega\|
$$

Hence, operator $\mathscr{P}$ is an isometric isomorphic mapping.

Then, we demonstrate $Y$ that is uniformly convex. For $\forall \tilde{\varepsilon}>0, \omega, v \in Y$, such that $\|\omega\|=\|v\|=1,\|\omega-v\| \geq \tilde{\varepsilon}$, choos$\operatorname{ing} \tilde{\delta}(\tilde{\mathcal{\varepsilon}})=1-\sqrt{1-\tilde{\varepsilon}^{2} / 4}>0$. On account of Lemma 4 , particularly $p=2$, we deduce

$$
\left\|\frac{\omega+v}{2}\right\| \leq \sqrt{\frac{1}{2}\left(\|\omega\|^{2}+\|v\|^{2}\right)-\left\|\frac{\omega-v^{2}}{2}\right\|} \leq \sqrt{1-\frac{\tilde{\varepsilon}^{2}}{4}}=1-\tilde{\delta}(\tilde{\varepsilon}) .
$$

By Lemma 2, $Y$ is uniformly convex. Owing to $\mathscr{P}$ is an isometric isomorphic mapping, and $\mathscr{W}$ is also uniformly convex. Part 3. holds by Lemma 3.

We complete the proof of the theorem.

\section{Main Results}

In this section, we will show there are at least three solutions for problem (1) mainly by Theorem 6. Define function

$$
\rho(t, \eta)=\int_{0}^{\eta} g(t, s) d s
$$

for any $(t, \eta) \in[0,1] \times \mathbb{R}$, and $g:[0,1] \times \mathbb{R} \longrightarrow \mathbb{R}$ is a continuous function. For our main results, the following lemma is first given.

Lemma 8. Suppose that there exist six positive constants $K, P$, $L, A_{1}, A_{2}$ and $\widehat{A}$ with $P<L \sqrt{K} / 8 \widehat{A}$ and $0<A_{1}<A_{2}<1$, such that

(1) $\rho(t, \eta) \geq 0,(t, \eta) \in\left[0, A_{1}\right] \cup\left[A_{2}, 1\right] \times[0,(L / 4 \widehat{A})]$;

(2) $\max _{(t, \eta) \in[0,1] \times[-P, P]} \rho(t, \eta)<64 \widehat{A}^{2} P^{2} / K L^{2} \int_{A_{1}}^{A_{2}} \rho(t,(L / 4 \widehat{A})) d t$,

then, there exist $\alpha>0, \mu \in \mathscr{W}$ such that $2 \alpha<\|\mu\|^{2}$ and

$$
\max _{(t, \eta) \in[0,1] \times[-P, P]} \rho(t, \eta)<2 \alpha \frac{\int_{0}^{1} \rho(t, \mu(t)) d t}{\|\mu\|^{2}} .
$$

Proof. Define

$$
\mu(t)=L\left(t-\frac{1-\int_{0}^{1} t d \gamma(t)}{1-\int_{0}^{1} t^{2} d \gamma(t)} t^{2}\right) .
$$

Notice that space $\mathscr{W}$ is normed $\|\mu\|=\left\{\int_{0}^{1}\left|\mu^{\prime}\right|^{2} d t\right\}^{1 / 2}$. It is readily found that $\mu(t) \in \mathscr{W}$. In a simple calculation, we have

$$
\|\mu\|^{2}=\int_{0}^{1}\left|\mu^{\prime}\right|^{2} d t=L^{2}\left(1-\frac{10 \widehat{A}}{3}\right)^{2}
$$

where

$$
\widehat{A}=\frac{1-\int_{0}^{1} t d \lambda(t)}{1-\int_{0}^{1} t^{2} d \lambda(t)}
$$

By the hypotheses $P<L \sqrt{K} / 8 \widehat{A}$, choosing $\alpha=2 P^{2}, 0<$ $A_{1}<1 / 2 \widehat{A}<A_{2}<1$, any number and $K=16(3-10 \widehat{A})^{2} \widehat{A}^{2} / 9$, we have $2 \alpha<\|\mu\|^{2}$. According to assumption (a), one has

$$
\begin{aligned}
2 \alpha \frac{\int_{0}^{1} \rho(t, \mu(t)) d t}{\|\omega\|^{2}} & \geq \frac{4 P^{2}}{\|\mu\|^{2}} \int_{A_{1}}^{A_{2}} \rho\left(t, \frac{L}{4 \widehat{A}}\right) d t \\
& =\frac{4 P^{2}}{L^{2}(1-10 \widehat{A} / 3)^{2}} \int_{A_{1}}^{A_{2}} \rho\left(t, \frac{L}{4 \widehat{A}}\right) d t \\
& =\frac{64 \widehat{A}^{2} P^{2}}{K L^{2}} \int_{A_{1}}^{A_{2}} \rho\left(t, \frac{L}{4 \widehat{A}}\right) d t \\
& >\max _{(t, \eta) \in[0,1] \times[-P, P]} \rho(t, \eta) .
\end{aligned}
$$

Thus, inequality (29) holds.

Theorem 9. Assume that there exist eight positive constants $K$, $P, L, A_{1}, A_{2}, c, \kappa$, and $\widehat{A}$ with $P<L \sqrt{K} / 8 \widehat{A}, 0<A_{1}<A_{2}<1$ and $\kappa<2$, such that

(1) $\rho(t, \eta) \geq 0,(t, \eta) \in\left[0, A_{1}\right] \cup\left[A_{2}, 1\right] \times[0,(L / 4 \widehat{A})]$;

(2) $\max _{(t, \eta) \in[0,1] \times[-P, P]} \rho(t, \eta)<64 \widehat{A}^{2} P^{2} / K L^{2} \int_{A_{1}}^{A_{2}} \rho(t,(L / 4 \widehat{A})) d t$;

(3) $\rho(t, \eta) \leq c\left(1+|\eta|^{\kappa}\right)$, for $t \in[0,1]$ and $\eta \in \mathbb{R}$

Then, there exists an open interval $\Omega \subseteq(0,+\infty)$ and $a$ positive real number $p$ such that for each $\beta \in \Lambda$, problem (1) has at least three solutions belonging to $\mathscr{W}$ whose norms are less than $p$. 
Proof. Consider the following functions.

$$
\begin{aligned}
2 \alpha & \frac{\int_{0}^{1} \rho(t, \mu(t)) d t}{\|\omega\|^{2}} \geq \frac{4 P^{2}}{\|\mu\|^{2}} \int_{A_{1}}^{A_{2}} \rho\left(t, \frac{L}{4 \widehat{A}}\right) d t \\
& =\frac{4 P^{2}}{L^{2}(1-10 A \wedge / 3)^{2}} \int_{A_{1}}^{A_{2}} \rho\left(t, \frac{L}{4 \widehat{A}}\right) d t \\
& =\frac{64 A \wedge^{2} P^{2}}{K L^{2}} \int_{A_{1}}^{A_{2}} \rho\left(t, \frac{L}{4 \widehat{A}}\right) d t>\max _{(t, \eta) \in[0,1] \times[-P, P]} \rho(t, \eta) .
\end{aligned}
$$

for any $\omega \in \mathscr{W}$.

Notice that the critical points of $\Gamma$ are the generalized solutions of problem (1). Therefore, we just validate that $Y$ and $\Phi$ accord with the conditions of Theorem 6 . It is obvious that $Y$ is a continuously Gâteaux differentiable and sequentially weakly lower semicontinuous functional whose Gâa teaux derivative yields a continuous inverse on $\mathscr{W}^{*}$, and $\Phi$ is a cntinuously Gâteaux differentiable functional whose Gâa teaux derivative is compact.

In addition, by condition (c) and Poincare' inequality, one readily has

$$
\lim _{\|\omega\| \rightarrow+\infty} \Gamma(\omega)=\lim _{\|\omega\| \rightarrow+\infty} Y(\omega)+\beta \Phi(\omega)=+\infty
$$

for any $\beta \geq 0$.

Next, we prove that there exist $\alpha>0, \omega \in \mathscr{W}$ satisfying

$$
\sup _{\omega \in \Phi^{-1} \hat{\mathscr{E}}(-\infty, \alpha] \in \odot}(-\Phi(\omega))<-\alpha \frac{\Phi(\omega)}{Y(\omega)}
$$

From Lemma 8, $\omega(t)=\mu(t)$, one has $\max _{t \in[0,1]}|\omega(t)|=L$ $14 \widehat{A}$; on the other hand, $\|\omega\|=|L(1-10 \widehat{A} / 3)|$. Thus, $\max _{t \in[0,1]}|\omega| \leq\|\omega\|$, for all $\omega \in \mathscr{W}$. Thus,

$$
Y^{-1}(-\infty, \alpha] \subseteq\{\omega \in \mathscr{W}:\|\omega\| \leq \sqrt{2 \alpha}, 0 \leq t \leq 1\}
$$

for any $\alpha>0$. Moreover,

$\sup _{\omega \in Y^{-1}(-\infty, \alpha]}(-\Phi(\omega)) \leq \sup _{\|\omega\|^{2} \leq 2 \alpha} \int_{0}^{1} \rho(t, \omega) d t \leq \max _{(t, \eta) \in[0,1] \times\left[-\sqrt{\frac{\alpha}{2}}, \sqrt{\frac{\alpha}{2}}\right]} \rho(t, \eta)$.

From Lemma 8, one has

$$
\max _{(t, \eta) \in[0,1] \times\left[-\sqrt{\frac{\alpha}{2}}, \sqrt{\frac{\alpha}{2}}\right]} \rho(t, \eta)<2 \alpha \frac{\int_{0}^{1} \rho(t, \omega(t)) d t}{\|\omega\|^{2}}=-\alpha \frac{\Phi(\omega)}{Y(\omega)} .
$$

So, inequality (36) holds.
By Lemma 5 , selecting $H(\beta)=\rho \beta$, we have

$\sup _{\beta \geq 0} \inf _{\omega \in \mathscr{W}}\left(Y(x)+\beta(\Phi(x)+\rho)<\inf _{\omega \in \mathscr{W}} \sup _{\beta \geq 0}(Y(x)+\beta(\Phi(x)+\rho)\right.$.

Therefore, the proof is complete by Theorem 6 .

For the purpose of explaining the validity of our results, an example is given as follows.

Example. Consider the special second-order problem as below.

$$
\left\{\begin{array}{l}
-\omega^{\prime \prime}(t)=\beta t f(\omega) \\
\omega(0)=0, \omega(1)=\int_{0}^{1} \omega(s) d s
\end{array}\right.
$$

where $g(t, \omega)=t f(\omega), \quad \gamma(s)=s$. Choosing $L=30, f(\omega)$ defined by

$$
f(\omega)= \begin{cases}e^{\omega}, & \text { if } \omega \leq \frac{L}{3}, \\ \omega+e^{L / 3}-\frac{L}{3}, & \text { if } \omega>\frac{L}{3} .\end{cases}
$$

Proof. According to the integrability of $f(\omega)$ and $f(\omega) \geq 0$ for $\omega \in[0, \eta]$, where $\eta \in[0,(L / 4 \widehat{A})]$, we have

$$
\int_{0}^{\eta} f(y) d y \geq 0
$$

which means $t \int_{0}^{\eta} f(y) d y \geq 0$, for $t \in\left[0, A_{1}\right] \cup\left[A_{2}, 1\right]$. So, the assumption (a) of Theorem 9 is satisfied.

Define

$$
F(\eta)=\int_{0}^{\eta} f(y) d y
$$

Thus,

$$
F(\eta)=\left\{\begin{array}{lr}
e^{\eta}-1, & \text { if } \eta \leq \frac{L}{3}, \\
\eta\left(e^{L / 3}-\frac{L}{3}\right)+e^{L / 3}\left(1-\frac{L}{3}\right)+\frac{L^{2}}{18}+\frac{\eta^{2}}{2}-1, & \text { if } \eta>\frac{L}{3} .
\end{array}\right.
$$

Owing to $g(t, \omega)=t f(\omega)$, one has

$$
\max _{(t, \eta) \in[0,1] \times[-P, P]} \rho(t, \eta) \leq \max _{(t, \eta) \in[0,1] \times[-P, P]} \int_{0}^{\eta} g(t, y) d y=\max _{t \in[0,1]} t F(P) .
$$

Choosing $\kappa=3 / 2, c=e^{L / 3}, P=1, K=9, A_{2}=1 / 2$, and $A_{2}$ $=3 / 4$, we guarantee that conditions (b) and (c) of Theorem 9 are satisfied. 


\section{Data Availability}

No data were used to support the findings of study.

\section{Conflicts of Interest}

The authors declare that they have no conflicts of interest.

\section{Acknowledgments}

This research is supported by the NSFC of China (62073203) and the Fund of Natural Science of Shandong Province (ZR2018MA022).

\section{References}

[1] P. Amster and C. Rogers, "On Dirichlet two-point boundary value problems for the Ermakov-Painlevé IV equation," Journal of Applied Mathematics and Computing, vol. 48, no. 1-2, pp. 71-81, 2015.

[2] Z. Cen, L. B. Liu, and J. Huang, "A posteriori error estimation in maximum norm for a two-point boundary value problem with a Riemann-Liouville fractional derivative," Applied Mathematics Letters, vol. 102, p. 106086, 2020.

[3] Y. Niu and B. Yan, "The existence of positive solutions for the singular two-point boundary value problem," Topological Methods in Nonlinear Analysis, vol. 48, no. 1, pp. 1-682, 2017.

[4] Y. Niu and B. Yan, "Exact number of positive solutions for a class of two-point boundary value problems," Journal of Applied Mathematics, vol. 2013, Article ID 589483, 8 pages, 2013.

[5] C. Xenophontos, "Isogeometric analysis for singularly perturbed high-order, two-point boundary value problems of reaction-diffusion type," Computers and Mathematics with Applications, vol. 80, no. 11, pp. 2340-2350, 2020.

[6] M. F. Aktaş, D. Çakmak, and A. Tiryaki, "On the Lyapunovtype inequalities of a three-point boundary value problem for third order linear differential equations," Applied Mathematics Letters, vol. 45, pp. 1-6, 2015.

[7] H. Djourdem and S. Benaicha, "Existence of positive solutions for s nonlinear three-point boundary value problem with integral boundary conditions," Acta Mathematica Universitatis Comenianae, vol. 87, pp. 167-177, 2018.

[8] Y. H. Zhang, Z. B. Bai, and T. T. Feng, "Existence results for a coupled system of nonlinear fractional three-point boundary value problems at resonance," Computers \& Mathematics with Applications, vol. 61, no. 4, pp. 1032-1047, 2011.

[9] Y. Liu, "Solvability of multi-point boundary value problems for multiple term Riemann- Liouville fractional differential equations," Computers \& Mathematics with Applications, vol. 64, no. 4, pp. 413-431, 2012.

[10] S. Smirnov, "Green's function and existence of solutions for a third-order three-point boundary value problem," Mathematical Modelling and Analysis, vol. 24, no. 2, pp. 171-178, 2019.

[11] Q. H. Zhang, Y. Wang, and Z. M. Qiu, "Existence of solutions and boundary asymptotic behavior of $p(r)$-Laplacian equation multi-point boundary value problems," Nonlinear Analysis: Theory, Methods \& Applications, vol. 72, no. 6, pp. 29502973, 2010.

[12] B. Ahmad and S. K. Ntouyas, "Existence results for nonlocal boundary value problems of fractional differential equations and inclusions with strip conditions," Boundary Value Problems, vol. 2012, no. 1, Article ID 145, 2012.

[13] J. R. Cannon and A. L. Matheson, "A numerical procedure for diffusion subject to the specification of mass," International Journal of Engineering Science, vol. 31, no. 3, pp. 347-355, 1993.

[14] J. R. Cannon, L. Yanping, and W. Shingmin, "An implicit finite difference scheme for the diffusion equation subject to mass specification," International Journal of Engineering Science, vol. 28, no. 7, pp. 573-578, 1990.

[15] H. Cheng and R. Yuan, "Existence and asymptotic stability of traveling fronts for nonlocal monostable evolution equations," Discrete \& Continuous Dynamical Systems - B, vol. 22, no. 7, pp. 3007-3022, 2017.

[16] H. Cheng and R. Yuan, "Existence and stability of traveling waves for Leslie-Gower predator-prey system with nonlocal diffusion," Discrete \& Continuous Dynamical Systems - A, vol. 37, no. 10, pp. 5433-5454, 2017.

[17] H. Cheng and R. Yuan, "Traveling waves of some HollingTanner predator-prey system with nonlocal diffusion," Applied Mathematics and Computation, vol. 338, no. 1, pp. 12-24, 2018.

[18] Y. Liu and D. O'Regan, "Controllability of impulsive functional differential systems with nonlocal conditions," Electronic Journal of Differential Equations, vol. 2013, no. 194, pp. 1-10, 2013.

[19] G. L. Karakostas and P. C. Tsamatos, "Existence of multiple positive solutions for a nonlocal boundary value problem," Topological Methods in Nonlinear Analysis, vol. 19, no. 1, pp. 109-121, 2002.

[20] B. Yan, "Positive solutions for the singular nonlocal boundary value problems involving nonlinear integral conditions," Boundary Value Problems, vol. 2014, no. 1, Article ID 38, 2014.

[21] B. Yan, D. O'Regan, and R. P. Agarwal, "Multiplicity and uniqueness results for the singular nonlocal boundary value problem involving nonlinear integral conditions," Boundary Value Problems, vol. 2014, no. 1, Article ID 148, 2014.

[22] H. Cheng and R. Yuan, "The stability of the equilibria of the Allen-Cahn equation with fractional diffusion," Applicable Analysis, vol. 98, no. 3, pp. 600-610, 2017.

[23] Y. Liu and H. Yu, "Bifurcation of positive solutions for a class of boundary value problems of fractional differential inclusions," Abstract and Applied Analysis, vol. 2013, Article ID 942831, 8 pages, 2013.

[24] Y. Liu, "Positive solutions using bifurcation techniques for boundary value problems of fractional differential equations," Abstract and Applied Analysis, vol. 2013, Article ID 162418, 7 pages, 2013.

[25] Y. Liu, "Bifurcation techniques for a class of boundary value problems of fractional impulsive differential equations," Journal of Nonlinear Science and Application, vol. 8, pp. 340-353, 2015.

[26] K. N. O, K. S. Jong, S. A. Pak, and H. C. Choi, "A new approach to approximate solutions for a class of nonlinear multi-term fractional differential equations with integral boundary conditions," Advances in Difference Equations, vol. 2020, no. 1, Article ID 2739, 2020.

[27] Q. L. Song and Z. B. Bai, "Positive solutions of fractional differential equations involving the Riemann-Stieltjes integral boundary condition," Advances in Difference Equations, vol. 2018, no. 1, Article ID 1633, 2018. 
[28] Y. Sun and M. Zhao, "Positive solutions for a class of fractional differential equations with integral boundary conditions," Applied Mathematics Letters, vol. 34, pp. 17-21, 2014.

[29] X. G. Zhang, L. S. Liu, B. Wiwatanapataphee, and Y. H. Wu, "The eigenvalue for a class of singular $p$-Laplacian fractional differential equations involving the Riemann-Stieltjes integral boundary condition," Applied Mathematics and Computation, vol. 235, pp. 412-422, 2014.

[30] H. Zhang, "Iterative solutions for fractional nonlocal boundary value problems involving integral conditions," Boundary Value Problems, vol. 2016, no. 1, 2016.

[31] I. Belmouloud and A. Memou, "On the solvability of a class of nonlinear singular parabolic equation with integral boundary condition," Applied Mathematics and Computation, vol. 373, article 124999, 12 pages, 2020.

[32] B. Abdelkader, "Second-order boundary value problems with integral boundary conditions," Nonlinear Analysis, vol. 70, no. 1, pp. 364-371, 2009.

[33] M. Benchohra, J. J. Nieto, and A. Ouahab, "Second-order boundary value problem with integral boundary conditions," Boundary Value Problems, vol. 2011, Article ID 260309, 9 pages, 2011.

[34] M. M. Chaharlang, M. A. Ragusa, and A. Razani, "A Sequence of Radially Symmetric Weak Solutions for Some Nonlocal Elliptic Problem in $R^{N}$," Mediterranean Journal of Mathematics, vol. 17, no. 2, article 1492, pp. 1-12, 2020.

[35] M. A. Ragusa and A. Razani, "Weak solutions for a system of quasilinear elliptic equations," Contributions to Discrete Mathematics, vol. 1, pp. 11-16, 2020.

[36] A. Razani, "An existence theorem for ordinary differential equation in Menger probabilistic metric space," Miskolc Mathematical Notes, vol. 15, no. 2, pp. 711-716, 2014.

[37] A. Razani, "Structure of shock wave for a viscous combustion model," Filomat, vol. 26, no. 2, pp. 389-395, 2012.

[38] A. Razani, "Chapman-Jouguet travelling wave for a two-steps reaction scheme," Italian Journal of Pure and Applied Mathematics, vol. 39, pp. 544-553, 2018.

[39] R. Y. Ma, "Positive solutions for second order functional differential equations," Dynmatic Systems and Applications, vol. 10, pp. 215-223, 2001.

[40] J. Zhang, G. Zhang, and H. Li, "Positive solutions of secondorder problem with dependence on derivative in nonlinearity under Stieltjes integral boundary condition," Electronic Journal of Qualitative Theory of Differential Equations, vol. 2018, no. 4, pp. 1-13, 2018.

[41] J. Galvis, E. M. Rojas, and A. V. Sinitsyn, "Existence of positive solutions of a nonlinear second-order boundary-value problem with integral boundary conditions," Electronic Journal of Differential Equations, vol. 2015, no. 236, pp. 1-7, 2015.

[42] X. Li and S. Song, "Stabilization of delay systems: delaydependent impulsive control," IEEE Transactions on Automatic Control, vol. 62, no. 1, pp. 406-411, 2017.

[43] X. Li and J. Wu, "Stability of nonlinear differential systems with state-dependent delayed impulses," Automatica, vol. 64, pp. 63-69, 2016.

[44] J. Mawhin and M. Willem, Critical Ponints Theory and Hamitonian Systems, Springer-Verlag, Berlin, 1989.

[45] B. Ricceri, "Existence of three solutions for a class of elliptic eigenvalue problems," Mathematical and Computer Modelling, vol. 32, no. 11-13, pp. 1485-1494, 2000.
[46] B. Ricceri, "On a three critical points theorem," Archiv der Mathematik, vol. 75, no. 3, pp. 220-226, 2000.

[47] C. E. Lawrence, "Partial differential equations," American Mathematical Society Providence, vol. 19, 2010. 